\title{
Study on the one-dimensional carriage and ventilation system of high- speed train
}

\author{
Yifei Zhu, Yugong Xu, Xiangdong Chen \\ School of Mechanical Electronic and Control Engineering, Beijing Jiao tong University, Beijing \\ 100044, China
}

Keywords: high-speed train, one-dimensional, carriage, ventilation system, simulation.

\begin{abstract}
Interior and external environment of the high-speed train are connected by the ventilation system, but the structure of the complex ventilation system and slenderness is relatively large, and therefore can be a one-dimensional assumption. This paper selects IC06 carriage of high-speed train CRH380B EMU as the research. The results calculated were compared with the design, and the one-dimensional modelling method is reasonable. The use of the one-dimensional simulation proved by means of high-speed train carriage and ventilation system to study reliability, so you can use the modelling approach other conditions to predict the environmental conditions inside the train carriages.
\end{abstract}

\section{Introduction}

Interior environment of the high-speed train is to contact the external environment through the ventilation system, and the current interior environmental studies of high-speed train are mostly separate. The carriage as a research object, given the carriage inlet and outlet boundary conditions for numerical simulation [1-3], which ignores the influence of the ventilation system of the interior environment, reducing the accuracy of the results. However, the composition of the complex ventilation system, air inlet, grill, controllable dampers, ducts, air conditioning units, air orifice, return air valves, waste discharge unit like a series of complex parts [4,5], these three-dimensional structures are built mild and simulation would require huge computing resources can be achieved only in such a case, taking into account the efficiency of research, and in the case the length is much larger than the hydraulic diameter of the ventilation duct, the one-dimensional ventilation system of the high-speed train will be a high accuracy[6], and thus the carriage and ventilation system were one-dimensional model, numerical simulation research has become an important research tool. This paper selects carriage IC06 of the high-speed train CRH380B EMU as an object of study, according to the model to determine the one-dimensional simulation of the desired geometric parameters and technical parameters based on one-dimensional simulation software FLOWMASTER established carriage and ventilation system analysis model through simulation and with the design results were compared to verify the reasonableness and accuracy of the onedimensional model, which is used to prove that the use of a one-dimensional simulation by means of high-speed train carriage and ventilation system reliability study.

\section{FLOWMASTER computing introduction}

FLOWMASTER conduct in the use of simulation, fluid grid can be seen as a series of fluid piping components, component model is mainly based on the pressure - flow relationship. Between piping components connected by nodes. Mobile network in the various components of the conservation of mass and energy conservation is given the export algebraic equation to describe. These algebraic equations generally $\mathrm{f}$ (pressure, flow) $=0$ for linear equations. That is, the fluid in the pipe network components is seen as resistance members, each pipe and valves equivalent impedance positive effect. Pumps fans and other negative effects of the equivalent impedance, all elements of resistance can be determined according to the corresponding flow, pressure head or power factor, such a fluid network simplifies problem solving a system of linear equations has become a problem. 
FLOWMASTER solving is based matrix solver to solve the entire model. As shown in Fig.1, for a simple network diagram.

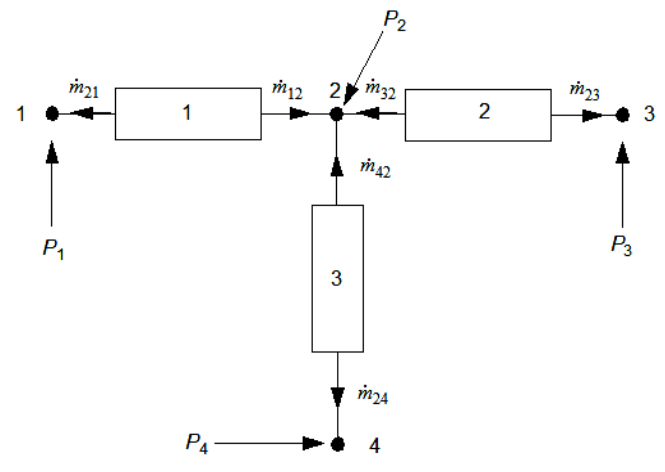

Fig. 1 Matrix solver schematic

Fig. 1 for the network model, its analysis matrix [7]:

Wherein, in the matrix of coefficients is determined by the parameters of the corresponding elements. When the entire network system to solve the model will be used to set the initial flow is solved, when solved after completion of the first, got the pressure of each node, and then the pressure can be obtained by a new flow, therefore, the matrix each factor will be modified to be solved again. For the solution process the entire network model, this is through an iterative process to achieve reciprocation until all the results have reached the preset time residuals. At last the calculation is complete.

$$
\begin{aligned}
& {\left[\begin{array}{cccc}
a_{21}^{1} & a_{22}^{1} & 0 & 0 \\
a_{11}^{1} & a_{21}^{1}+a_{21}^{3}+a_{21}^{3} & a_{11}^{2} & a_{11}^{3} \\
0 & a_{22}^{2} & a_{21}^{2} & 0 \\
0 & a_{22}^{3} & 0 & a_{21}^{3}
\end{array}\right]\left[\begin{array}{c}
P_{1} \\
P_{2} \\
P_{3} \\
P_{4}
\end{array}\right]=} \\
& {\left[\begin{array}{c}
\dot{m}_{21}-a_{23}^{1} \\
\dot{\cdot} \\
m_{12}+m_{32}+m_{42}-a_{13}^{1}-a_{13}^{2}-a_{13}^{3} \\
\dot{m_{23}}-a_{23}^{2} \\
\dot{m_{24}}-a_{23}^{3}
\end{array}\right]}
\end{aligned}
$$

\section{The carriage and ventilation system model}

Fig.2 shows the high-speed train from CRH380B models IC06 carriage looks relatively smooth, only the air conditioning unit was outstanding shape. There can be seen the type of carriage a combination of fresh air ventilation system parts, exhaust section and return air section together. New air vents and grille section includes a new, pressure protection valves, mixing box, air conditioning units, the new wind blowing Road, spoiler, air porous roof, windows and under the foot outlet and other components; where the air conditioning unit structure shown in Figure1, includes an evaporator, an expansion valve, an electric heater, two fan assembly, air filter, air pressure switches and other components. Section includes vehicle exhaust air vents and grilles, exhaust duct, waste discharge unit, air vents and grille. Partly because of the return air and is directly connected to the mixing tank, only controlled by an electric valve, no duct, simple structure, as shown in Fig.2.

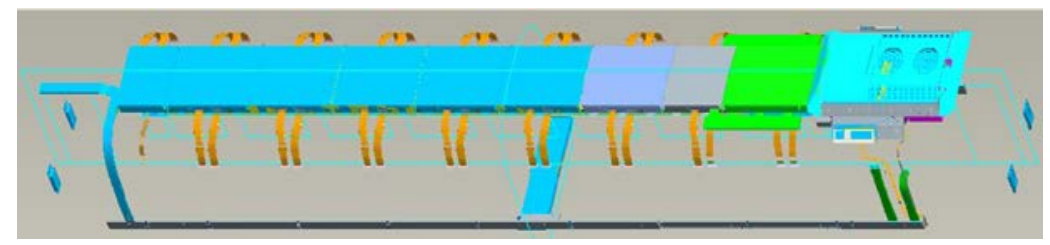

Fig.2 Ventilation system diagram of carriage IC06 
Because the model is too complicated, too much components, parameter settings uncertainty is too large, in order to make the system modeling process from modeling process piping affect change, and to simplify the system, without affecting the calculation made on the basis of some necessary simplification.

1) CRH380B models of pressure protection valve has only two modes is open or closed, in normal operation, the valve is always open, so you can ignore the part in the establishment of a vascular network systems.

2) Air conditioning mixing box is just a confluence of container shaped like a Y-pipe, the drag coefficient is small, and therefore considered as a modeling point of convergence.

3) Because they do not analyze the changes of temperature field inside, so you can simplify the modeling part of refrigeration and air conditioning, the air conditioning system air filters, electric heaters, evaporators reduced to simple resistance element is calculated based on local resistance:

Get the air filter is calculated according to local resistance, local resistance evaporator, fan heaters, heater duct silencers, air conditioning units and other components added resistance.

The formula is:

$$
Z=\xi \frac{v^{2} \rho}{2}
$$

Unit: Pa.

Where: $\zeta$-- local resistance coefficient, look-up table can be obtained.

$\rho-$ - Fluid density in $\mathrm{kg} / \mathrm{m} 3$.

$\mathrm{v}--$ Flow velocity in $\mathrm{m} / \mathrm{s}$.

The total resistance of the pipe section is provided local resistance $\mathrm{Z}$, is calculated using the lcoal resistance coefficient:

$$
\xi^{\prime}=\frac{2 Z}{v^{2} \rho}
$$

4) The supply duct system lines appearing in the majority of the exhaust pipe is a straight pipe, is only a few individual bends and corners very small, which produces additional resistance relative to the entire pipe network resistance is quite small, So the elbow ventilation ducts are omitted meter, are used instead of straight pipe.

5) For the pipeline, in connection with the main duct drainage play the role of spoiler fresh air, so it can be simplified to the resistance elements.

6) For the plates, as well as modeling the window slit outlet, have simplified plate model, its parameter settings only need to enter the actual area and the effective orifice area, so the closest outlet to send the real situation.

Ventilation systems and air-conditioning system for the main components of the waste exhaust fan unit. Intake and exhaust fans are centrifugal fans, model shown in Fig.3.

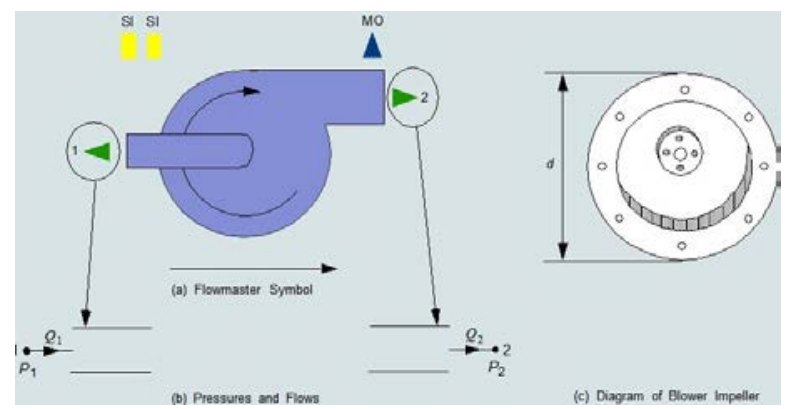

Fig.3 Simplified model of centrifugal fan

$\mathrm{d}$ = diameter of the impeller, Q1 = inlet volume flow, $\mathrm{Q} 2=$ export volume flow, $\mathrm{P} 1=$ centrifugal fan inlet pressure, $\mathrm{P} 2$ = outlet pressure centrifugal blower.

In FLOWMASTER, the description for setting the fan performance indicators is included more than a few. In the selection of performance indicators used in the calculation, including fan wind, air pressure, effective power, shaft power, efficiency of the fan and fan speed. In addition, the characteristic curve of the fan is also a major factor affecting the performance of the fan, the fan 
provides air pressure (blower and export full pressure) should be able to call the loss of resistance along the way, and local resistance losses in the entire ventilation system air flow generated Fig.4 and Fig.5 shown the inlet and exhaust fan characteristic curve.

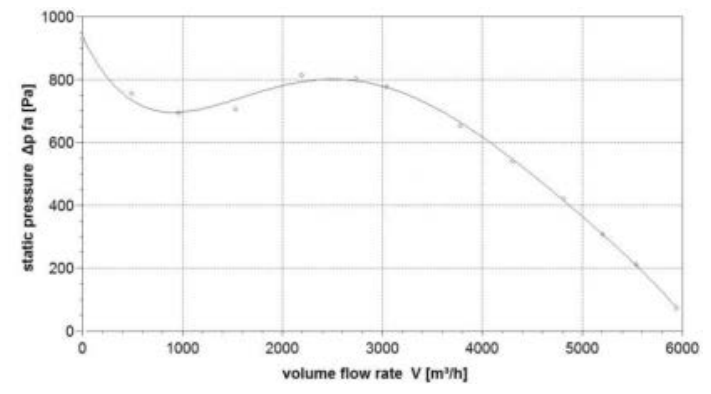

Fig.4 Characteristic curve of intake fan

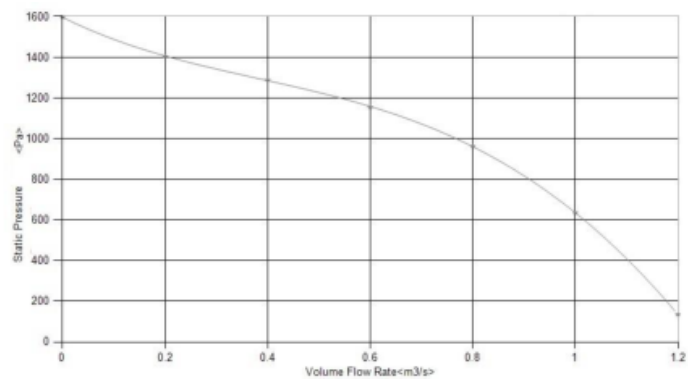

Fig.5 Characteristic curve of exhaust fan

CRH380B models based on real data of IC06 carriage and ventilation system as a data base, the establishment of a one-dimensional high-speed train carriage and ventilation system pipe network system as shown in Fig.6.

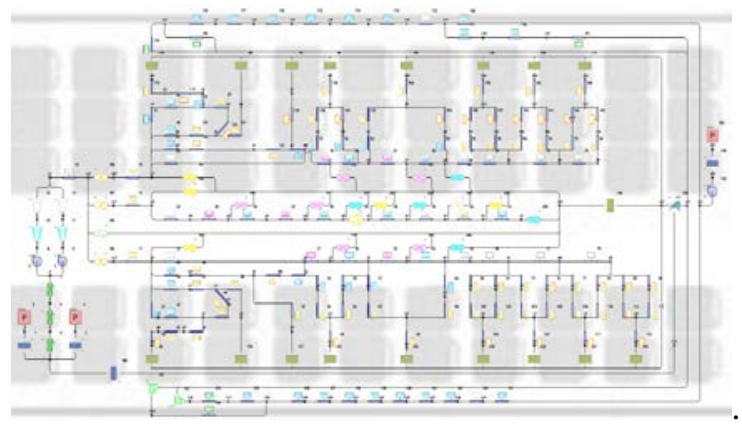

Fig.6 Carriage and ventilation systems model

\section{Conclusions}

Results of a vascular network system debugging requirements in one atmospheric environment, the new outlet flow, exhaust port flow, return air flow and air flow rate of each branch to meet design conditions. . Shown in two new air inlet flow were $0.1789 \mathrm{~m} 3$ / s, the main air duct flow $1.2794 \mathrm{~m} 3$ / $\mathrm{s}$, the exhaust port flow $0.3571 \mathrm{~m} 3 / \mathrm{s}$, the calculation results are consistent with the actual operating point of the ventilation system, and data such as shown in Tab.1.

According to the results, the model parameter set reasonable, the results and the real operating point of the ventilation system are consistent, which demonstrates the reasonableness and accuracy of the simulation model. So we can use the modeling approach other conditions to predict the environmental conditions inside the train carriage. 
Tab.1 The contrast table of the simulation results and the design working condition

\begin{tabular}{ccc}
\hline Parameters & Design working condition & Simulation results \\
\hline $\begin{array}{c}\text { Fresh air volume } \\
(\mathrm{m} 3 / \mathrm{h})\end{array}$ & 1200 & 1288.08 \\
$\begin{array}{c}\text { Air inlet volume } \\
(\mathrm{m} 3 / \mathrm{h})\end{array}$ & 4500 & 4605.84 \\
$\begin{array}{c}\text { Air outlet volume } \\
(\mathrm{m} 3 / \mathrm{h})\end{array}$ & 1200 & 1285.56 \\
$\begin{array}{c}\text { Return air volume } \\
(\mathrm{m} 3 / \mathrm{h})\end{array}$ & 3300 & 3320 \\
$\begin{array}{c}\text { Air supply velocity } \\
(\mathrm{m} / \mathrm{s})\end{array}$ & $2 \sim 5$ & 2.5912 \\
\hline
\end{tabular}

\section{Acknowledgements}

This work is supported by grants from the national 863 project No.2011AA11A1 03, and he Fundamental Research Funds for the Central Universities No.2012JB M093. The authors are grateful for this financial support.

\section{References}

[1] Berlitz, T. and G. Matched, Interior air flow simulation in railway rolling stock. Proceedings of the Institution of Mechanical Engineers, Part F: Journal of Rail and Rapid Transit, 2002. 216(4): p. 231-236.

[2] Kuang, X., et al., LES of airflow organization in the passenger compartments of the airconditioned high-speed train. Journal of the China Railway Society, 2009, 31(3): p. 96-99.

[3] Cao, X., et al., Numerical simulation and optimization for the air conditioning and ventilation system in the compartment of urban rail train. China Railway Science, 2013, 34(6): p. 105-109.

[4] DONG Ximing. High speed EMU working principle and structural characteristics. Beijing, China Railway Press, 2007.

[5] Liu Gang. The Numerical Simulation of the Train’s Air-conditioning Ventilation System. Da Lian Jiao Tong University, 2008.

[6] H.D. Kim, Y.K. Eum, K. Matsuo. One-dimensional numerical study of compression wave propagating in high-speed railway tunnel [J]. KSME 1995, 19 (5): p.1280 1290.

[7] FLOWMASTER V7 software module and its new features .CAD / CAM and manufacturing information 2008 (12): p.34-37. 